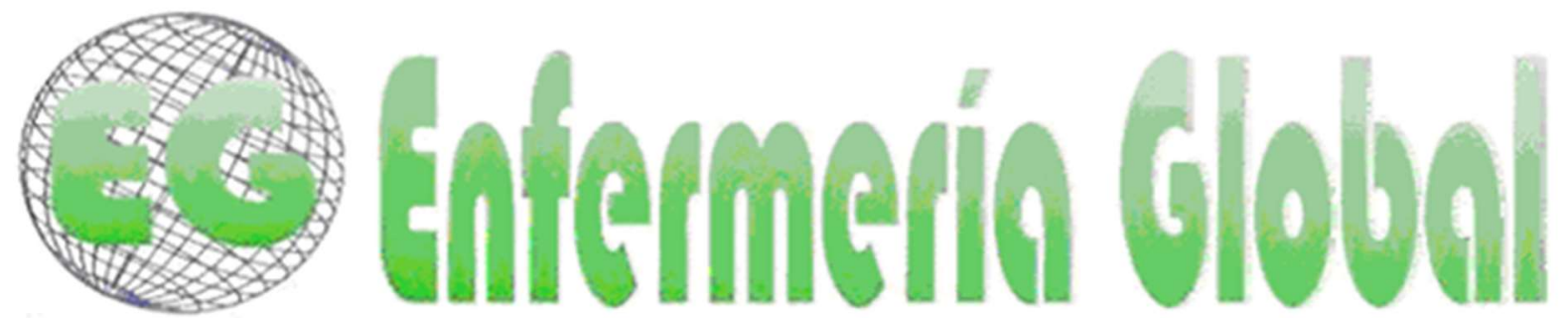

\title{
ORIGINALES
}

\section{Caída y factores demográficos y clínicos en adultos mayores: estudio de seguimiento}

Queda e fatores demográficos e clínicos no idoso: estudo de seguimento

Fall and demographic and clinical factors in older adults: follow-up study

Jack Roberto Silva Fhon ${ }^{1}$

Rosalina Aparecida Partezani Rodrigues ${ }^{2}$

${ }^{1}$ Doctor en Ciencias. Profesor del Departamento de Médico-Quirúrgica de la Escuela de Enfermería de la Universidad de São Paulo. Brasil. betofhon@usp.br

2 Doctora en Enfermería. Profesora Titular del Departamento de Enfermería General y Especializada de la Escuela de Enfermería de Ribeirão Preto de la Universidad de São Paulo. Brasil.

\section{https://doi.org/10.6018/eglobal.418881}

Recibido: $17 / 03 / 2020$

Aceptado: 4/07/2020

\section{RESUMEN:}

Objetivo: Analizar los factores demográficos y clínicos relacionados a las caídas en el adulto mayor que viven en el domicilio en un seguimiento de cinco años

Método: Estudio cuantitativo y longitudinal retrospectivo realizado entre 2007/2008 y 2013 con 262 participantes brasileños con edad superior a 65 años. Se utilizaron los instrumentos de perfil demográfico, Mini Examen del Estado Mental, enfermedades autorreferidas, caídas y sus características, Escala de Fragilidad de Edmonton, Escala de Lawton y Brody y Medida de Independencia Funcional. Se realizaron análisis descriptivo, Chi cuadrado, prueba de Wilcoxon y prueba de modelos mixtos. Estudio aprobado por el comité de ética.

Resultados: La mayoría era del sexo femenino, con edad entre 65 a 79 años, escolaridad entre 1 a 4 años y jubilados. La prevalencia de caídas fue de $21,8 \%$ y $37,8 \%$ en ambas evaluaciones. Se verificó que para cada enfermedad más que el adulto mayor tiene, aumenta la chance de sufrir una nueva caída. Además, para cada punto más en la escala de fragilidad, el adulto mayor tiene mayor chance de caer. Por otro lado, para cada medicamento a menos que el adulto mayor utiliza, presenta $10 \%$ menos chance de caer.

Conclusión: La caída estuvo asociado a factores clínicos en el adulto mayor siendo necesario que el profesional de salud realice evaluaciones constantes para identificar este evento y sus desencadenantes.

Palabras clave: Anciano; Accidentes por caídas; Fragilidad; Estudios longitudinales; Personal de salud; Consecuencias de accidentes.

\section{RESUMO:}

Objetivo: Analisar os fatores demográficos e clínicos relacionados à queda no idoso que vive no domicílio em um seguimento de cinco anos.

Método: Estudo quantitativo e longitudinal retrospectivo realizado entre 2007/2008 e 2013 com 262 participantes brasileiros com idade superior de 65 anos. Foram utilizados os instrumentos de perfil 
demográfico, Mini Exame do Estado Mental, doenças autorreferidas, quedas e suas características, Escala de Fragilidade de Edmonton, Escala de Lawton y Brody y Medida de Independência Funcional. Realizaram-se análise descritivo, Qui quadrado, Teste de Wilcoxon e prova de modelos mistos. Estudo aprovado pelo comitê de ética.

Resultados: A maioria era do sexo feminino, com idade entre 65 e 79 anos, escolaridade entre 1 a 4 anos e aposentados. A prevalência da queda foi de $21,8 \%$ e $37,8 \%$ em ambas as avaliações. Verificouse que para cada doença a mais que o idoso sofre, aumenta a chance de sofrer uma nova queda. Ademais, para cada ponto a mais na escala de fragilidade, o idoso apresenta uma maior chance de cair. Por outro lado, para cada medicamento a menos que o idoso utiliza, presenta chance $10 \%$ menor de cair.

Conclusão: A queda esteve associada a fatores clínicos no idoso sendo necessário que o profissional de saúde realize avaliações constantes com a finalidade de identificar esse evento e seus desencadeantes.

Palavras-chave: Idoso; Acidentes por quedas; Fragilidade; Estudos longitudinais; Pessoal de saúde; Consequências de acidentes.

\begin{abstract}
:
Objective: To analyze the demographic and clinical factors related to falls of older adults living at home in a five-year follow-up

Materials and methods: Retrospective quantitative and longitudinal study conducted between 2007/2008 and 2013 with 262 Brazilians participants over 65 years old. We used the demographic profile, Mini-Mental State Examination, self-reported diseases, falls and their characteristics, Edmonton Frail Scale, Lawton and Brody Scale, Functional Independence Measure instruments. We performed descriptive analysis, Chi-squared, Wilcoxon test and mixed model test.

Results: Most participants were women, aged between 65 to 79 years, schooling between 1 to 4 years and retired. The prevalence of falls was $21.8 \%$ and $37.8 \%$ in both evaluations. We verified that the chance of suffering a new fall increases for each additional disease of the older adult. In addition, for each point to more on the frail scale, the older adult has a greater chance of falling. On the other hand, for each medication withdrawn, the chance of falling decreases $10 \%$.

Conclusion: The fall was associated with clinical factors in older adults, therefore constant evaluations must be carried out to identify this event and its triggers.
\end{abstract}

Keywords: Aged; Accidental falls; Frailty; Longitudinal studies; Health personnel; Accident consequences

\title{
INTRODUCCIÓN
}

En el mundo se observa un aumento de la expectativa de vida y del número de personas con 60 años y más de edad. Datos del World Population Prospects muestran que en 2019 una de cada 11 personas en el mundo tenía más de 65 años $(9 \%)$ y se prevé que para 2050 será una de cada seis $(16 \%)$. Además, se prevé que el número de personas con 80 años y más se triplique, de 143 millones en 2019 a 426 millones en 2050 (1).

Durante el proceso de envejecimiento ocurren cambios fisiológicos que llevan al adulto mayor a sufrir de múltiples síndromes, entre ellos la caída. La Organización Mundial de la Salud (OMS) refiere que es la segunda causa de muerte por lesiones no accidentales, siendo un problema de salud pública, estimándose 646,000 caídas fatales por año en el mundo ${ }^{(2)}$.

La prevalencia de caídas puede afectar aproximadamente del $24 \%$ al $50 \%$ de los adultos mayores con edad superior a 60 años ${ }^{(3)}$. Además, en los Estados Unidos cada 20 minutos un adulto mayor sufre una caída (4) causando un gasto de $\$ 50$ billones de dólares en costos médicos para las caídas no fatales y $\$ 754$ millones de dólares para las fatales ${ }^{(5)}$. 
En Brasil, el Departamento de Informática del Sistema Único de Salud - DATASUS, reportó que entre los años 2007 y 2017 este evento fue la causa de muerte de 11,078 individuos diagnosticados según el ClE-10, en el grupo etario mayores de 60 años. Se identificó que hubo 7,996 óbitos, siendo que entre 60 y 69 años fueron 1,192, de 70 a 79 de 2,023 y mayores de 80 años de 4,781 muertes ${ }^{(6)}$.

La caída puede ser resultado de la interacción de los factores intrínsecos que son definidos como aquellos que están relacionados con el propio individuo ${ }^{(7)}$, además de la presencia de enfermedades agudas o crónicas y factores extrínsecos ${ }^{(8)}$. En la literatura es identificado que la mayor parte de las caídas ocurre durante las actividades rutinarias ${ }^{(7)}$ y la caída recurrente puede llevarlo a sufrir el síndrome del miedo a nuevas caídas ${ }^{(9)}$.

La caída puede ser ocasionada por el propio proceso de envejecimiento vinculado a múltiples factores que pueden llevar al adulto mayor a una incapacidad parcial o total que puede perjudicar su autonomía e independencia. El profesional de salud debe realizar un acompañamiento a los adultos mayores para detectar los factores que desencadenan la caída con la finalidad de realizar un planeamiento y aplicación de las medidas que las evite, para eso el estudio tuvo como objetivo principal analizar los factores demográficos y de salud relacionados a las caídas en el adulto mayor que viven en el domicilio en un seguimiento de cinco años.

\section{MÉTODO}

Estudio longitudinal retrospectivo realizado entre octubre de 2007 y marzo de 2008 (T1) y julio a diciembre de 2013 (T2) en la ciudad de Ribeirão Preto, São Paulo, Brasil. La obtención de la muestra fue por medio del modelo conglomerado probabilístico realizado en dos etapas. Para la primera fue considerado el sector censitario como la Unidad Primaria de la Muestra (UPM), en que los 600 sectores de la ciudad fueron sorteados, siendo 30 que tuvieron la probabilidad proporcional al tamaño del número de los domicilios. En el segundo fueron sorteadas las calles y cuadras de los sectores indicados en cada sector.

Los errores de la muestra fueron fijados en torno del $10 \%$ y para prevalencias de caídas de hasta el $70 \%$. Intervalos de confianza para prevalencias estimadas en estratos posteriores definidos por sexo y edad, considerándose el $10 \%$ como el límite máximo como error tolerado teniendo como muestra final 515 participantes.

Los criterios de inclusión en el T1 fueron ser adultos mayores con edad igual o superior a 65 años, de ambos sexos, capaces de comunicarse verbalmente y que vivan en la zona urbana y en el T2 fue haber participado de la primera evaluación.

En el T1 la recolección de los datos fue realizada en sentido horario y en el T2 se realizó el contacto telefónico con el adulto mayor o el familiar para agendar una visita en el domicilio. En aquellos adultos mayores en que no fue posible el contacto telefónico, fue necesario el desplazamiento de los investigadores a la dirección para programar una visita.

Se aplicó un instrumento para identificar los datos personales, las enfermedades autorreferidas, el número de medicamentos según la última receta médica, las caídas 
en los últimos 12 meses identificando el número de caídas, sus características, causas, consecuencias físicas, psicológicas y sociales.

El Mini Examen del Estado Mental (MEEM) traducido y validado para la lengua portuguesa (10). El score varía de 0 a 30 y los puntos de corte fueron 20 para no escolarizados, 24 para aquellos entre 1 a 4 años, 26,5 con 5 a 8 años, 28 entre 9 a 11 años y 29 para aquellos con escolaridad superior a 11 años.

La Medida de Independencia Funcional (MIF) validada ${ }^{(11)}$ para el portugués. Entre las actividades evaluadas están el desempeño para la realización de 18 actividades en conjunto referentes al autocuidado, transferencia, locomoción, control de esfínteres, comunicación y cognición social que incluye la memoria, interacción social y resolución de problemas presentando un score de 18 a 126 puntos.

La Escala de Lawton y Brody identifica las Actividades Instrumentales de la Vida Diaria (AIVD), validado para el contexto brasileño ${ }^{(12)}$. Presenta un score de siete a 21 puntos caracterizando al participante en dependencia total, dependencia parcial e independencia.

La Escala de Fragilidad de Edmonton (EFS) creada por el grupo Canadian Initiative on Frailty and Aging (CIF-A), validada y reproducida para Brasil (13). La escala evalúa nueve dominios representado en 11 ítems y presenta un score de cero a 17 puntos, siendo que cuanto mayor la puntuación en la escala, mayor es el nivel de fragilidad.

Para el análisis de las informaciones fue utilizado el programa Statistical Package for the Social Sciences - SPSS v. 25.0. Se utilizó la estadística descriptiva siendo que para las variables cuantitativas la medida de tendencia central (media) y de dispersión (desviación estándar) y para las categóricas frecuencias y porcentajes.

Se realizó la prueba de normalidad para las variables cuantitativas tal como EFS, MIF, AIVD, enfermedades autorreferidas y número de medicamentos, al no identificarse la normalidad fue utilizado la Prueba no paramétrica de Wilcoxon. Para las variables categóricas fue utilizado a Prueba de Chi cuadrado.

En el análisis final, la variable caída fue dividida en sí y no y las variables exploratorias que entraron en el modelo final fueron sexo (masculino y femenino), estado conyugal dividido en con compañero (casado) y sin compañero (soltero, viudo y divorciado) y o tiempo, en este caso, el año 2013 y entre las variables de salud las enfermedades autorreferidas, el número de medicamentos, score de la EFS, MIF y AIVD. Para todas las pruebas estadísticas se tuvo una significancia de $p \leq 0,05$.

El estudio fue aprobado por el Comité de Ética en Investigación de la Escuela de Enfermería de Ribeirão Preto de la Universidad de São Paulo, para el T1 el protocolo $\mathrm{n}^{\circ} 0851 / 2007$ y para el T2 el protocolo $\mathrm{n}^{\circ} 1392 / 2011$

\section{RESULTADOS}

En el T1, la muestra fue compuesta de 515 participantes y para el T2 fueron encontrados 262. Entre las perdidas en el curso de los cinco años se verificó que 127 
$(24,7 \%)$ fallecieron, $83(16,1 \%)$ rechazaron participar en el T2, $8(1,6 \%)$ fueron institucionalizados y $35(6,8 \%)$ se mudaron para otras ciudades.

En relación al perfil demográfico en el T2, fue identificado que la mayoría era del sexo femenino, con edad entre 65 a 79 años, escolaridad entre 1 a 4 años y jubilados. Además, hubo predominio de los viudos (Tabla 1).

Tabla 1: Perfil demográfico del adulto mayor que vive en el domicilio. Ribeirão Preto, Brasil, 2019.

\begin{tabular}{|c|c|c|c|c|}
\hline \multirow[b]{2}{*}{ Variables } & \multicolumn{4}{|c|}{$2013(n=262)$} \\
\hline & Categorías & $\mathbf{n}$ & $\%$ & Media (=DE) \\
\hline \multirow[t]{2}{*}{ Sexo } & Masculino & 88 & 33,6 & \\
\hline & Femenino & 174 & 66,4 & \\
\hline \multirow[t]{2}{*}{ Edad } & $65-79$ & 149 & 56,9 & $79,3(6,34)$ \\
\hline & 80 y más & 113 & 43,1 & \\
\hline \multirow[t]{5}{*}{ Estado civil } & Viudo & 116 & 44,3 & \\
\hline & Casado & 106 & 40,5 & \\
\hline & Soltero & 25 & 9,5 & \\
\hline & Divorciado & 12 & 4,6 & \\
\hline & Separado & 3 & 1,1 & \\
\hline \multirow[t]{5}{*}{ Escolaridad } & No escolarizado & 45 & 17,2 & $5,00(4,95)$ \\
\hline & $1-4$ & 139 & 53,1 & \\
\hline & $5-8$ & 33 & 12,6 & \\
\hline & $9-11$ & 9 & 3,4 & \\
\hline & 12 y más & 36 & 13,7 & \\
\hline \multirow[t]{2}{*}{ Jubilado } & $\mathrm{Si}$ & 176 & 67,4 & \\
\hline & No & 86 & 32,6 & \\
\hline
\end{tabular}

En relación a la caída, de los 262 adultos mayores, la prevalencia en el T1 fue de $21,8 \%$ y en el T2 de $37,8 \%$. Se verificó que en el T1 y T2 hubo predominio de aquellos que sufrieron apenas una caída (Tabla 2).

Tabla 2: Prevalencia y número de caídas en el adulto mayor que vive en el domicilio en el seguimiento 2007/2008 - 2013. Ribeirão Preto, Brasil, 2019.

\begin{tabular}{lcccc}
\hline & \multicolumn{2}{c}{$\mathbf{2 0 0 7 / 2 0 0 8}$} & \multicolumn{2}{c}{$\mathbf{2 0 1 3}$} \\
\multicolumn{1}{c}{ Variables } & $\mathbf{n}$ & $\mathbf{\%}$ & $\mathbf{n}$ & $\mathbf{\%}$ \\
\hline Prevalencia de caída & 57 & 21,8 & 99 & 37,8 \\
Número de caídas & & & & \\
1 & 33 & 57,9 & 67 & 67,7 \\
$2-4$ & 17 & 29,8 & 29 & 29,3 \\
$\geq 5$ & 7 & 12,3 & 3 & 3,0 \\
\hline
\end{tabular}

En el análisis bivariada, en el T1 se verificó asociación entre la caída con las AIVD, fragilidad y número de enfermedades. En el T2, la caída se asoció a la fragilidad, número de enfermedades y al uso de medicamentos (Tabla 3 ). 
Tabla 3: Asociación entre caídas con las variables demográficas y de salud en el adulto mayor que vive en el domicilio. Ribeirão Preto, Brasil, 2019.

\begin{tabular}{|c|c|c|c|c|c|c|}
\hline \multirow[b]{2}{*}{ Variable } & \multicolumn{3}{|c|}{$\begin{array}{c}2007 / 2008 \\
\text { Caída }\end{array}$} & \multicolumn{3}{|c|}{$\begin{array}{l}2013 \\
\text { Caída }\end{array}$} \\
\hline & $\begin{array}{c}\mathbf{S i} \\
\mathrm{n}(\%)\end{array}$ & $\begin{array}{c}\text { No } \\
n(\%)\end{array}$ & $\mathbf{p}$ & $\begin{array}{c}\mathbf{S i} \\
\mathrm{n}(\%)\end{array}$ & $\begin{array}{c}\text { No } \\
\text { n (\%) }\end{array}$ & $\mathbf{p}$ \\
\hline Estado cognitivo & & & & & & \\
\hline Sin déficit & $27(18,6)$ & $118(81,4)$ & 0,171 & $54(35,3)$ & $99(64,7)$ & $0,324^{*}$ \\
\hline $\begin{array}{l}\text { Con déficit } \\
\text { AIVD }\end{array}$ & $30(26,6)$ & $87(74,4)$ & & $45(41,3)$ & $64(58,7)$ & \\
\hline Independencia & $24(16,6)$ & $121(83,4)$ & 0,023 & $32(36,0)$ & $57(64,0)$ & $0,661^{*}$ \\
\hline $\begin{array}{l}\text { Dependencia } \\
\text { MIF }\end{array}$ & $33(28,2)$ & $84(71,8)$ & & $67(38,7)$ & $106(61,3)$ & \\
\hline Independencia & $53(21,1)$ & $198(78,9)$ & 0,230 & $79(35,4)$ & $144(64,6)$ & $0,060^{*}$ \\
\hline Dependencia & $4(36,4)$ & $7(63,4)$ & & $20(51,3)$ & $19(48,7)$ & \\
\hline Fragilidad & $4,98( \pm 2,40)$ & $3,93( \pm 2,63)$ & $0,002^{*}$ & $7,49( \pm 3,10)$ & $5,94( \pm 2,94)$ & $<0,001^{\dagger}$ \\
\hline Número de enfermedades & $6,74( \pm 3,52)$ & $5,32( \pm 3,43)$ & $0,007^{*}$ & $6,01( \pm 3,45)$ & $4,64( \pm 3,46)$ & $0,002 \dagger$ \\
\hline Número de medicamentos & $4,18( \pm 2,58)$ & $3,43( \pm 2,75)$ & $0,068^{*}$ & $4,07( \pm 3,23)$ & $4,01(3,52)$ & $0,893 \dagger$ \\
\hline
\end{tabular}

Al comparar las características de la caída, en el T1, 82,5\% fue de la propia altura, $57,9 \%$ usaban medicamentos, 40,4\% presentaron alteración del equilibrio, $45,6 \%$ uso de zapatos inadecuados, $24,6 \%$ cayeron en el patio y $43,9 \%$ presentaron miedo de sufrir una nueva caída.

En el T2, 73,7\% cayeron de la propia altura, $26,3 \%$ usaban algún tipo de medicamento, 30,3\% tuvieron alteración del equilibrio, 37,4\% usaban zapatos inadecuados, $18,2 \%$ indicaron que cayeron en el patio de la casa y $39,4 \%$ refirieron tener miedo de caer (Tabla 4).

Tabla 4: Características de la caída y sus consecuencias en el adulto mayor que vive en el domicilio. Ribeirão Preto, Brasil, 2019.

\begin{tabular}{llcccc}
\hline \multirow{2}{*}{ Características } & \multicolumn{1}{c}{ Variables } & \multicolumn{2}{c}{$\mathbf{2 0 0 7 / 2 0 0 8}$} & \multicolumn{2}{c}{$\mathbf{2 0 1 3}$} \\
\hline Altura de la caída & Propia altura & $\mathbf{n}$ & $\mathbf{\%}$ & $\mathbf{n}$ & $\mathbf{\%}$ \\
& Escalera & 47 & 82,5 & 73 & 73,7 \\
& Sillón & 7 & 12,3 & 5 & 5,1 \\
& Cama & 3 & 5,3 & 5 & 5,1 \\
& Silla para tomar baño & - & - & 9 & 9,1 \\
Uso de medicamentos & & - & - & 4 & 4,0 \\
Factores intrínsecos & Alteración del equilibrio & 33 & 57,9 & 26 & 26,3 \\
& Dificultad de caminar & 23 & 40,4 & 30 & 30,3 \\
& Mareos & 7 & 12,3 & 13 & 13,1 \\
& Confusión mental & 6 & 10,5 & 5 & 6,1 \\
& Debilidad muscular & 4 & 7,0 & 1 & 1,0 \\
Factores extrínsecos & 3 & 5,3 & 10 & 10,2 \\
& Desmayo & 3 & 5,3 & 3 & 3,0 \\
& Zapato inadecuado & 26 & 45,6 & 37 & 37,4 \\
& Piso resbaladizo & 14 & 24,6 & 15 & 15,2 \\
& Piso irregular & 9 & 15,8 & 10 & 10,1 \\
& Desnivel de escalones & 8 & 14,0 & 5 & 5,1 \\
& Objetos en el piso & 3 & 5,3 & 7 & 7,1 \\
& Animales & 3 & 5,3 & 1 & 1,0 \\
& Subir en objetos & 3 & 5,3 & 2 & 2,0 \\
\hline
\end{tabular}




\begin{tabular}{llcccc}
\hline & Baño sin apoyo & 2 & 3,5 & 1 & 1,0 \\
& Ropas grandes & 2 & 3,5 & 2 & 2,0 \\
& Tapetes & 2 & 3,5 & 2 & 2,0 \\
& Accesorios de apoyo & 1 & 1,8 & 6 & 6,1 \\
& lluminación inadecuada & 1 & 1,8 & 1 & 1,0 \\
& Patio & 14 & 24,6 & 18 & 18,2 \\
& Vereda & 12 & 21,1 & 13 & 13,1 \\
& Calle & 10 & 17,5 & 14 & 14,1 \\
& Baño & 9 & 15,8 & 12 & 12,1 \\
& Cocina & 8 & 14,0 & 11 & 11,1 \\
& Sala & 5 & 8,8 & 14 & 14,1 \\
& Dormitorio & 4 & 7,0 & 12 & 12,1 \\
& Hall de entrada & 3 & 5,3 & 1 & 1,0 \\
& Miedo de caer & 25 & 43,9 & 39 & 39,4 \\
& Afecto al caminar & 11 & 19,3 & 14 & 14,1 \\
& Ayuda en la rutina & 6 & 10,5 & 8 & 8,1 \\
& Ansiedad & 3 & 5,3 & 4 & 4,0 \\
& Perdida para organizar su vida & 2 & 3,5 & 1 & 1,0 \\
\hline
\end{tabular}

En la regresión de modelos mixtos se observó que el participante tiene mayor chance de sufrir caídas en el T2 comparado con el T1. En relación al número de enfermedades, fue verificado que para cada enfermedad más que sufre el participante, aumenta la chance de sufrir una nueva caída.

Además, para cada punto más en la EFS, el adulto mayor tiene mayor chance de caer. Por otro lado, para cada medicamento menos que el adulto mayor utiliza, presenta $10 \%$ menos chance de caer (Tabla 5).

Tabla 5: Regresión de modelos mixtos entre la caída y variables demográficas y de salud del adulto mayor. Ribeirão Preto, Brasil, 2019.

\begin{tabular}{lccccc}
\hline \multicolumn{1}{c}{ Variables } & B & Std Error & t-valor & OR (IC 95\%) & p \\
\hline Tiempo 2013 & 0,6877 & 0,2509 & 2,741 & $1,989[1,216-3,252]$ & 0,006 \\
Número medicamentos & $-0,1043$ & 0,0494 & $-2,11$ & $0,901[0,817-0,992]$ & 0,034 \\
Número enfermedades & 0,1222 & 0,0450 & 2,715 & $1,130[1,034-1,234]$ & 0,006 \\
Fragilidad & 0,1720 & 0,0479 & 3,591 & $1,187[1,081-1,304]$ & $<0,001$ \\
\hline
\end{tabular}

OR = Odds Ratio; IC = Intervalo de Confianza; $p<0,05$

\section{DISCUSIÓN}

En el estudio se verificó que la ocurrencia de caídas en el adulto mayor aumenta con el transcurso del tiempo, al aumento del número de enfermedades, y presencia de fragilidad. Además, el usar menos medicamentos, disminuye la chance de caer.

La caída es la causa más frecuente de lesiones en el adulto mayor, especialmente en aquellos considerados los más viejos, llevándolo a una disminución de la funcionalidad y muerte. En este estudio, la prevalencia de caídas en el T1 fue de $21,8 \%$ y aumentó en el T2 para $37,8 \%$. Un estudio americano analizó la prevalencia de este evento entre 1988 y 2010, los autores identificaron que en la primera evaluación la prevalencia de caídas fue de $28,2 \%$ y en la segunda de $36,3 \%{ }^{(14)}$. 
En otro estudio realizado en Inglaterra en el domicilio, se identificó una prevalencia de caídas en los últimos dos años de $28,4 \%{ }^{(15)}$. El aumento de este evento se debe al incremento de la expectativa de vida y la realización de actividades en el hogar y en la calle ${ }^{(16)}$.

La caída está vinculada a alteraciones de la postura y a la gravedad que es importante para mantener el equilibrio postural (17). Además, del sistema nervioso que regula el sistema músculo-esquelético garantizando el control de la postura y que en el proceso de envejecimiento se pierde ${ }^{(18)}$.

En el estudio se identificó que en ambas evaluaciones la caída fue de la propia altura, uso de zapatos inadecuados, el lugar más común de la caída fue el patio y presentaron miedo de caer. Investigaciones con resultados similares fueron realizadas en el Perú (3) y Estados Unidos ${ }^{(19)}$.

La caída de la propia altura es un evento no intencional que tiene como resultado el cambio de posición del individuo para un nivel inferior en relación a su posición inicial( $^{(20)}$ y que las lesiones, en la mayoría de las veces, pueden pasar desapercibidas por el profesional de salud.

El uso de calzado inadecuado se ha identificado como un contribuyente de hasta el $45 \%$ de las caídas (21). Asimismo, caminar descalzo o en medias también puede aumentar este riesgo. Además, se ha demostrado que usar zapatos tipo botas o con tacón alto aumenta el riesgo y perjudica el equilibrio ${ }^{(22)}$.

Por otro lado, el miedo de sufrir una nueva caída es un fenómeno psicológico que lleva a estados de ansiedad, síntomas depresivos, estrés psicológico (23), disminución o perdida de la independencia funcional lo que causa aislamiento social en el adulto mayor ${ }^{(3)}$.

En el estudio se verificó que el adulto mayor presenta una mayor chance de caer cuando hay un aumento del número de enfermedades crónicas y al consumo de medicamentos. La tasa de crecimiento de la población con 60 años y más es cerca de tres veces mayor en los países menos desarrollados y eso se ve reflejado con la disminución de la mortalidad y mejora de la expectativa de vida. Sin embargo, ese crecimiento aumenta las tasas de prevalencia de enfermedades no transmisibles e incapacidad (24).

De acuerdo con el Prescribing Cost Analysis en Inglaterra, el número total de medicamentos dispensados en 2015 fue de 1,08 billones correspondiendo en media a 19,9 medicamentos por paciente ${ }^{(25)}$. Un estudio con 5213 adultos mayores, fue identificado que 1611 usaban más de cuatro medicamentos por día siendo que 569 reportaron haber sufrido caídas. Además, aquellos considerados en polifarmacia sufrieron $18 \%$ más caídas comparados con los no considerados en polifarmacia y los que usan más de 10 medicamentos presentan una tasa de $50 \%$ de sufrir caídas ${ }^{(26)}$.

Los adultos mayores que usan antidepresivos, sedativos o hipnóticos y antiepilépticos son más propensos a sufrir caídas recurrentes. Además, la polifarmacia aumenta la posibilidad de 1,5 a 2 veces para caídas recurrentes en los adultos mayores considerados más viejos ${ }^{(27)}$. 
En relación a la fragilidad fue verificado que el adulto mayor presenta mayor chance de caer. Una revisión sistemática con meta-análisis identificó 19 estudios longitudinales y en la evaluación de estos fue identificada una asociación entre la caída y la fragilidad (OR 1,80; IC 95\%, 1,51-2,13) ${ }^{(28)}$. Además, otro estudio identificó que los factores que influencian las caídas en los adultos mayores frágiles son presentar mareos, dificultad para caminar y el miedo de caer ${ }^{(7)}$.

La fragilidad y las caídas son síndromes con afecciones de salud multifactoriales que ocurren cuando los efectos acumulados del déficit de diferentes sistemas hacen que la persona sea más vulnerable. Ambos comparten los mecanismos fisiopatológicos, factores de riesgo y son el resultado de eventos adversos ${ }^{(29)}$. Un estudio chino con 500 adultos mayores frágiles, los autores verificaron una prevalencia de $41.5 \%$ de caídas con predominio del sexo femenino y en aquellos con 80 años y más, causando daños graves ${ }^{(30)}$.

Entre las limitaciones, está relacionado al número de pérdidas durante el seguimiento, sin embargo, los datos muestran la importancia de una mirada diferenciada por el equipo multiprofesional de salud. Otra está relacionada con la evaluación de la caída realizada en dos periodos con intervalos de cinco años lo que dificulta un análisis más detallado.

Los resultados muestran la importancia de la evaluación del adulto mayor por el profesional de salud para identificar la caída y los factores demográficos y clínicos asociados a este evento como el síndrome de la fragilidad.

\section{CONCLUSIÓN}

En este estudio se identificó que el adulto mayor aumenta el riesgo de sufrir una caída cuando está asociado a la fragilidad y número de enfermedades. Sin embargo, la chance de caer disminuye a cada medicamento menos.

Estudios longitudinales son fundamentales en la evaluación multidimensional del adulto mayor, lo que indica la importancia de evaluación por parte del equipo multiprofesional de salud. Es necesario inversiones de los gobiernos locales para la prevención de las caídas, así como al desarrollo de investigaciones para la prevención, tratamiento curativo y de rehabilitación, con la necesidad de reducir la incapacidad y consecuentemente la pérdida de la independencia.

\section{REFERENCIAS}

1. United Nation. Ageing. 2019. Fecha de consulta: 12 de diciembre de 2019. Disponible en: https://www.un.org/en/sections/issues-depth/ageing/

2. World Health Organization. Falls. 2018. Fecha de consulta: 5 de diciembre de 2019. Disponible en: http://www.who.int/news-room/fact-sheets/detail/falls

3. Silva-Fhon JR, Partezani-Rodrigues R, Miyamura K, Fuentes-Neira W. Causas y factores asociados a las caídas del adulto mayor. Enfermería Universitaria. 2019;16(1):31-40.

4. Center for Diseases Control and Prevention. Older adult falls. 2016. Fecha de consulta: 8 de diciembre de 2019. Disponible en: https://www.cdc.gov/homeandrecreationalsafety/falls/index.html) 
5. Florence CS, Bergen G, Atherly A, Burns E, Stevens J, Drake C. Medical costs of fatal and nonfatal falls in older adults. J Am Geriatr Soc. 2018;66(4):693-698.

6. Sistema DATASUS. Informações de saúde. 2019 Fecha de consulta: 20 de diciembre de 2019. Disponible en: http://tabnet.datasus.gov.br/cgi/tabcgi.exe?sim/cnv/obt10uf.def

7. Callis N. Falls prevention: identification of predictive falls risk factors. Appl Nurs Res. 2016;29:53-8.

8. Ravindran RM, Kutty VR. Risk factors for fall-related injuries leading to hospitalization among community-dwelling older persons: a hospital-based case-control study in Thiruvananthapuram, Kerala, India. Asia Pac J Public Health. 2016;28(1):70S-76S.

9. Tomita Y, Arima K, Tsujimoto R, Kawashiri SY, Nishimura T, Mizukami S, et al. Prevalence of fear of falling and associated factors among Japanese community-dwelling older adults. Medicine. 2018;97(4):e9721.

10. Brucki SMD, Nitrini R, Caramelli P, Bertolucci PHF, Okatomo IH. Sugestões para o uso do mini-exame do estado mental no Brasil. Arq Neuropsiquiatr. 2003;61(3-B):777-781.

11. Riberto M, Miyazaki MH, Jucá SSH, Sakamoto H, Pinto PPN, Battistella LR. Validação da versão brasileira da medida de independência funcional. Acta Fisiatr. 2004;11(2):72-76.

12. Santos RL, Virtuoso Júnior JS. Confiabilidade da versão brasileira da escala de atividades instrumentais da vida diária. RBPS. 2008;21(4):290-296.

13. Fabricio-Wehbe SCC, Cruz IR, Haas VJ, Diniz MA, Dantas RAS, Rodrigues RAP. Reproducibility of the Brazilian version of the Edmonton Frail Scale for elderly living in the community. Rev Latino-am Enfermagem. 2013;21(6):13306.

14. Cigolle CT, Há J, Min LC, Lee PG, Gure TR, Alexander NB, et al. The epidemiologic data on falls, 1998-2010: more older Americans report falling. JAMA Intern Med. 2015;175(3):443-445.

15. Gale CR, Cooper C, Sayer AA. Prevalence and risk factors for falls in older men and women: The English longitudinal study of ageing. Age ageing, 2016;45:789-794.

16. Pimentel WRT, Pagotto V, Stopa SR, Hoffmann MCCL, Andrade FB, Souza Junior PRB, et al. Quedas entre idosos brasileiros residentes em áreas urbanas: ELSI-Brasil. Rev Saude Publica. 2018;52(Supl 2):12s.

17. Cruz E, González M, López M, Godoy ID, Pérez MU. Caídas: revisión de nuevos conceptos. Revista HUPE. 2014;13(2):86-95.

18. Alfieri FM, Silva NOV, Kutz NA, Salgueiro MMHAO. Relações entre equilíbrio, força muscular, mobilidade funcional, medo de cair e estado nutricional de idosos da comunidade. Kairós Gerontologia. 2016;19(2):147-165.

19. Landers M, Oscar S, Sasaoka J, Vaughn K. Balance confidence and fear of falling avoidance behavior are most predictive of falling in older adults: prospective analysis. Phys Ther. 2016;96(4):433-442.

20. Moura RN, Santos FD, Driemeier M, Santos LD, Ramos LR. Quedas em idosos: fatores de risco associados. Gerontologia. 1999;7(2):15-21

21. Boelens C, Hekman EE, Verkerke GJ. Risk factors for falls of older citizens. Technol Health Care. 2013; 21(5): 521-33.

22. Van der Cammen TJM, Sterke CS, Halilovic A, Molenbroek J. Influence of footwear on gait characteristics that are associated with increaser falls risk in older persons. The ergonomics open journal, 2016;9:43-49. 
23. Santos SCA, Figueiredo DMP. Preditores do medo de cair em idosos portugueses na comunidade: um estudo exploratório. Ciên saúde coletiva. 2019;24(1):77-86.

24. Kampfen F, Wijemunige N, Evangelista Jr B. Aging, non-communicable diseases and old-age disability in low and middle-income countries: a challenge for global health. Int J Public Health. 2018;63(9):1011-1012.

25. Health \& Social Care Information Centre. Prescription Cost Analysis England 2015. 2016. Fecha de consulta: 30 de noviembre de 2019. Disponible en: https://files.digital.nhs.uk/publicationimport/pub20xxx/pub20200/pres-cost-anal-eng2015-rep.pdf

26. Dhalwani NN, Fahami R, Sathanapally H, Seidu S, Davies MJ, Khunti K. Association between polypharmacy and falls in older adults: a longitudinal study from England. BMJ Open. 2017;7:e016358.

27. Ming $Y$, Zecevic A. Medications and polypharmacy influence on recurrent faller in community: a systematic review. Can Geriatr J. 2018;21(1):14-25.

28. Fhon JRS, Rodrigues RAP, Neira WF, Huayta VMR, Robazzi MLCC. Queda e sua associação à síndrome da fragilidade no idoso: revisão sistemática com metanálise. Rev Esc Enferm USP. 2016;50(6):1005-1013.

29. Inouye SK, Studenski S, Tinetti ME, Kuchel GA. Geriatric syndromes: clinical, research, and policy implications of a core geriatric concept. J Am Geriatr Soc. 2007;55(5):780-791.

30. Pi HY, Hu MM, Jie Z, Peng PP, Nie D. Circumstances of falls and fall-related injuries among frail elderly under home care in China. International journal of nursing sciences, 2015;2(3):237-342.

ISSN 1695-6141

(c) COPYRIGHT Servicio de Publicaciones - Universidad de Murcia 